\title{
Bioengineered cell culture systems of central nervous system injury and disease
}

\section{Fábio G. Teixeira ${ }^{1,2}$, Natália L. Vasconcelos ${ }^{1,2}$, Eduardo D. Gomes ${ }^{1,2}$, Fernanda Marques ${ }^{1,2}$, João C. Sousa ${ }^{1,2}$, Nuno Sousa ${ }^{1,2}$, Nuno A. Silva ${ }^{1,2}$, Rita Assunção-Silva ${ }^{1,2}$, Rui Lima ${ }^{1,2}$ and António J. Salgado ${ }^{1,2}$}

\footnotetext{
${ }^{1}$ Life and Health Sciences Research Institute (ICVS), School of Health Sciences, University of Minho, Braga, Portugal

${ }^{2}$ ICVS/3B's, PT Government Associate Laboratory, Braga/Guimarães, Portugal
}

Cell culture systems, either 2D or explant based, have been pivotal to better understand the pathophysiology of several central nervous system (CNS) disorders. Recently, bioengineered cell culture systems have been proposed as an alternative to the traditional setups. These innovative systems often combine different cell populations in 3D environments that more closely recapitulate the different niches that exist within the developing or adult CNS. Given the importance of such systems for the future of CNS-related research, we discuss here the most recent advances in the field, particularly those dealing with neurodegeneration, neurodevelopmental disorders, and trauma.

\section{Introduction}

Injury and disease within the CNS frequently induce chronic and acute insults leading to irreversible processes resulting in neuronal cell death. This fact is intimately related to the low regenerative potential of the CNS and the complexity of its several niches. Additionally, the causes that induce such phenomena are multivariate in nature. Indeed, cell death within the CNS can be triggered by injury, as in traumatic brain injury (TBI) or spinal cord injury (SCI); protein aggregation, such as in the case of Alzheimer's (AD) or Parkinson's disease (PD); neurodevelopmental-related problems, such as Rett syndrome; or induced neurodevelopmental neurotoxicity (DNT) phenomena. Thus, understanding the mechanisms behind such pathologies, as well as the possible therapeutic strategies that could be used to counteract them, is essential. To do so, it is essential to understand how CNS cells operate under these conditions, as well as how they interact with each other.

Animal models of injury and disease, as well as CNS cell culture systems, are often use to assess CNS cell interactions and how they operate. Models of injury are often seen as the last phase of preclinical research and try to replicate, as far as possible, the

Corresponding author: Salgado, A.J. (asalgado@ecsaude.uminho.pt) molecular, biochemical, and phenotypical characteristics of the CNS condition under study. However, for the initial screening of therapies, such as a library of small molecules with potential therapeutic use, such models might not be the best option. For instance, the differences between animal and human cell biology can lead to misleading results in important areas, such as toxicology $[1,2]$. Models of disease have been also used for years to understand the molecular mechanisms of injury and disease of the CNS, as well as for the early development of therapeutic strategies. Within these models, cell lines such as SH-SY5Y [3], N2 [4] or PC-12 [5], or MO-4 [6] are commonly the first system to be used. However the gold standard within the field are primary cultures of CNS cells [neural stem cells (NSC), neurons, astrocytes, oligodendrocytes, and microglial cells], which are often isolated from embryonic or early postnatal [up to postnatal day (P)5] tissue from rodents, mainly mice and rats [7]. Other popular systems within the field are explant cultures [8] and organotypic-like cultures [9-11]. Explant cultures are often used to study, for example, the development of axons and processes associated with it, whereas organotypic-like cultures are popular in the study of how CNS cells work in a 3D environment, such as the spinal cord [9], hippocampus [10] or substantia nigra/striatum [11]. 
Although using such systems has resulted in many insights into how CNS cells operate, the fact is that they have several limitations. For instance, the primary tissue source for their isolation is rodent tissue, either from embryonic or early postnatal origin, which limits the extent of the studies that can be performed, namely if the goal is to study the disease and/or injury on human-based cell culture models for translational purposes. Moreover, the culture systems themselves do not exactly recapitulate the different niches of the CNS. For example, standard cell culture systems are often $2 \mathrm{D}$, whereas tissue structures are $3 \mathrm{D}$, and are limited to one or two cell types. In addition, both explant and organotypic culture systems are limited in time (e.g. 2 weeks), which is a disadvantage if the objective is to study protein aggregation, and disease progression, for example.

To overcome these limitations, different bioengineered systems have been proposed as reliable alternatives. Indeed, with the advent of stem cell biology, biomaterials, microfluidics, and robotics, new systems have been developed that more closely resemble the CNS environment. Thus, here we describe and discuss the most relevant and recent developments in this field (the most relevant of which are summarized in Table 1), particularly those related to brain barriers, neurodevelopmental disorders, differentiation, neurodegeneration, and trauma.

\section{Bioengineered systems in CNS injury and disease Brain barriers}

For CNS homeostasis, the existence of brain barriers is essential to actively limit and regulate the passage of cells and molecules into, and out of, the brain. Thus, the cells that constitute these barriers function not only as gatekeepers, but also to secrete specific molecules that influence brain physiology. The perturbation of brain barriers has been found in many neurological disorders, including those discussed here. Restoring the integrity of the blood-brain barrier (BBB) in pathological conditions to maintain brain homeostasis and, for instance, opening it temporarily to allow the efficient delivery of drugs to the CNS, are potential therapeutic options for patients with these disorders. Thus, the development of BBB models that can mimic as far as possible the environment observed in vivo are of the utmost interest in the field of CNS regenerative medicine.

There are two main brain barriers: the BBB and the bloodcerebrospinal fluid barrier (BCSFB). The meninges, which are also barriers within the brain, are not considered here [12]. The BBB is formed by the endothelial cells of blood capillaries, a basal lamina, pericytes, the end-feet of astrocytes, and a few neuronal terminals [13]. In turn, the main structure of the BCSFB is the choroid plexus (CP), which is a small membrane suspended inside the space of the CSF-filled brain ventricles. The key feature of the CP is a polarized monolayer of epithelial cells separated by tight junctions, with high secretory and/or transporter capacity; this layer separates the periphery (blood-side) on its basolateral side from the CNS (CSF side) on its apical side [14]. A well-recognized function of the $\mathrm{CP}$ is the production of CSF, which in turn is affected by alterations that occur in the CP epithelial cells [15]. Both the BBB and BCSFB are involved in several disorders of the CNS, including $\mathrm{AD}$, multiple sclerosis (MS), and stroke, prompting the need to develop in vitro cell culture systems that mimic the organization and functionality of these barriers.
In vitro $2 \mathrm{D}$ cell culture systems of the brain barriers are based on the principle of separation between two compartments, one that simulates the blood side and the other the brain parenchyma side. Separating these two compartments is a monolayer of endothelial cells (BBB) or CP epithelial cells (BCSFB) seeded on top of a usually porous filter, such as Transwell polycarbonate or polyethylene inserts. The establishment of the barrier properties of this in vitro model and, hence, of the absence of the paracellular passage of molecules, compounds, or cells, must be confirmed by, for example, the measurement of transendothelial (or epithelial) electrical resistance (TEER). This two-chambered, single monolayer, system is a less complex in vitro way of modeling the barriers in the brain. Although such models have some limitations, they are relevant for permeability screening, transport assays [16], and investigating the transcellular passage of immune cells, among other uses $[17,18]$. In the case of the BBB, co-culture and triple co-culture systems have been added to the monocultures, introducing astrocytes and/or pericytes to these systems in addition to the endothelial cells; although they better mimic the cellular organization of the BBB, these later cell culture systems are complex in terms of the culture medium conditions supplied to both compartments (extensively reviewed in [16]). Given the specific polarized nature of CP epithelial cells, with their apical side bathing the CSF, twocompartment CP cultures are used to test the effects of blood- [15] and brain parenchyma-derived molecules [19] on the barrier properties of the BCSFB.

Although the above cell culture systems have been extensively used to study the interplay between the CNS and the periphery, the need for further complex 3D culture systems that entirely mimic both the BBB and the BCSFB still exists, especially for human cells. A particular aspect in which bioengineered models could provide added value compared with more traditional coculture models, is in the use of flow to modulate the behavior of the cells. Indeed, shear stress, generated by the flow of blood under physiological conditions affects transporter as well as endothelial barrier function [20]. Endothelial cells and astrocytes are plated on the inner (luminal) and outer (abluminal) sides of porous hollow fibers, respectively, and the culture medium is then pumped into the system via a variable-speed pump to generate shear stress comparable with that seen in physiological conditions in vivo [21]. To maintain the stable microenvironment, a gas-permeable tubing system is used for the exchange of $\mathrm{O}_{2}$ and $\mathrm{CO}_{2}$. Further sideby-side comparative studies showed that this dynamic model generated a tighter barrier function [22]. Additionally, the expression of transporters, ion channels, and efflux proteins was dramatically induced in endothelial cells in this model. Altogether, these data suggest that this dynamic model better mimics the in vivo $\mathrm{BBB}$ by replicating its anatomic and physiological properties. This model has been used to study the pathophysiology of various CNS diseases, including epilepsy [23]. For instance, endothelial cells and astrocytes from normal or drug-resistant epileptic human brain tissue have been cultured using this dynamic system. The permeability to phenytoin, a substrate for P-glycoprotein, was significantly reduced when endothelial cells from epileptic brain were used. This effect was independent of the origin of astrocytes and could be reversed by the P-glycoprotein blocker XR9576, suggesting that the drug-resistant BBB phenotype in patients with epilepsy can be replicated using this dynamic BBB model. 
TABLE 1

Bioengineered systems in CNS research.

\begin{tabular}{|c|c|c|c|c|}
\hline Application & Concept & Materials & Cell types & Refs \\
\hline BBB & $\begin{array}{l}\text { Dynamic culture on porous hollow fibers } \\
\text { using flow to mimic shear stress. Culture } \\
\text { medium is pumped through the hollow } \\
\text { fiber to promote this condition }\end{array}$ & Polypropylene fibers & $\begin{array}{l}\text { Endothelial cells and astrocytes } \\
\text { are plated on the inner (luminal) } \\
\text { and outer (abluminal) sides of the } \\
\text { hollow fibers }\end{array}$ & {$[21-23]$} \\
\hline $\begin{array}{l}\text { BBB and } \\
\text { BCSFB }\end{array}$ & $\begin{array}{l}\text { Neurovascular unit on a chip using } \\
\text { microfluidics; comprises three PDMS layers }\end{array}$ & $\begin{array}{l}\text { PDMS (chambers); polycarbonate } \\
\text { (membranes); collagen (biomatrix }\end{array}$ & $\begin{array}{l}\text { Human endothelial cells, } \\
\text { pericytes, astrocytes, and neurons }\end{array}$ & {$[24,25]$} \\
\hline
\end{tabular}

Neurovascular unit on a chip using microfluidics; comprises three PDMS layers separated by $0.2-\mu \mathrm{m}$ pore membranes. Membranes are bonded between layers to recreate the microfluidic vasculature and brain compartments

\section{Mini brain} organoid Developmental brain model in which highdensity iPSCs are cultured for extended periods of time to enable the in vitro development of brain structures (membranes); collagen (biomatrix for nerve cells to grow in)

\section{CNS niche}

\section{Neuronal} networks
Establishment of ECM-like hydrogels that can support the growth of cells relevant for CNS regeneration

Printed 3D cell culture system

System that recapitulates CNS niche by mimicking layer-by-layer organization of the brain. Based on the combination of microchambers and biodegradable fibers

3D neural tissue-like structures with fully matured neuronal networks growing on hydrogels, interfacing with microelectrodes for cell stimulation and environment probing

Cell growth platforms developed using NSC and bioactive bioengineered surfaces obtained by microcontact printing, piezoelectric spotting of polycationic biomolecules or ECM on cell-repellent surfaces

\begin{tabular}{l} 
Cerebral ischemic \\
damage \\
\hline AD
\end{tabular}

Compartmentalized membrane system with a sandwich configuration

Long-term 3D high-density neuronal cell

cultures in matrigel to enable accumulation of amyloid- $\beta(A \beta)$ and hyperphosphorylated tau

\section{SCI}

In vitro model of SCl that replicates stereotypical cellular responses to neurological injury in vivo, including reactive gliosis, microglial infiltration, and limited nerve fiber outgrowth. Based on the combination of organotypical slices and nanofiber meshes Modeling isotropic-to-anisotropic cellular transfections observed in vivo following spinal cord injury. Comprises the combination of astrocytes and dissociated dorsal root ganglia on PLLA fibers and membranes
Long-term 3D-brain-like cortical tissues based on the combination of cells with $3 \mathrm{D}$ scaffolds and hydrogels subsequently exposed to a weight drop method injury
Gellan gum modified with cell adhesion-related motifs

Thermoresponsive biodegradable polyurethane bioink Collagen fibers layered on PDMS microchambers coated with polyL-lysine

Hydrogel capable of supporting neuronal cell growth (e.g., collagen, matrigel)

PEO; microelectrode arrays; cell adhesion peptides (e.g., RGD)
iPSCs as a starting point that then differentiate towards multiple CNS cell types reconstituting early phases of brain development, including formation of brain barrier-like structures

NSC; olfactory ensheathing cells; mesenchymal stem cells

NSC

Matured neurons

Mature neurons differentiated from NSC isolated from primary tissue or differentiated from iPSCs
NSC derived from human umbilical cord mononuclear cells
Fluorocarbon/polyethersulfone membranes

Matrigel

(n)

$\begin{aligned} & \text { Polylactic acid nanofiber meshes } \\ & \text { coated with laminin }\end{aligned}$
NSC

Neonatal rodent hippocampal cells and mesenchymal stem cells

Human neural progenitors

Poly-L-lactide (PLLA) fibers and membranes
Astrocytes and dissociated dorsal root ganglia
Hydrogel (collagen); 3D scaffolds (silk)
Rodent cortical neurons 
Other systems using different concepts, such as microfluidics, have also been presented. This particular technique has several advantages, such as the reduction in sample size and compartmentalization, which allows an accurate representation of different BBB and CNS niches, including the use of dynamic culturing conditions. The development of a complex 'neurovascular unit on a chip', simultaneously containing features of both the BBB and the BCSFB, has also been proposed [24]. Recently, the first neurovascular microfluidic reactor was developed and validated; this platform contains simultaneously human endothelial cells cultured with pericytes, astrocytes, and neurons embedded in extracellular matrix (ECM) components [25]. It comprises two chambers and three polydimethylsiloxane (PDMS) layers plus $0.2-\mu \mathrm{m}$ pore polycarbonate membranes between the two chambers. The membrane is bonded between layers of PDMS to create the microfluidic vasculature and brain compartments. The reactor also has two perfusion streams, one for the vascular media supply to the endothelial cells in layer one and another for the brain media supply to layers two and three, so that one can be used for the biomatrix (e.g., collagen) and cell loading, and the other for subsequent perfusion of the brain compartment. The reactor can be used to recreate important microenvironmental conditions, including cell/fluid volume ratios, spatial gradients, and proper fluid flows, which are particularly important for shear stresses conditions. Thus, this system, and others similar to it, will become an essential tool for understanding brain barriers under physiological and pathological scenarios, as well as how new drugs can modulate these barriers.

\section{Developmental disorders}

The development of the CNS is a tightly regulated process, and any interference in it, either from genetic or environmental factors, can result in severe learning and memory impairments, as well as motor and sensory dysfunctions [26]. Research on this topic is currently carried out by using models of developmental diseases, which can give insights into how a specific condition is established at the development stage or at a post-onset neurodegenerative stage, where the goal is to seek ways to potentiate the repair and regeneration of the damaged tissues or cells. Until a decade ago, the study of developmental disorders of the CNS was mainly based on genetic animal models, which, although they have contributed greatly to the advance of research in this field, they often present a phenotype that is very different from that of humans as a result of intraspecies variation [26]. As an alternative, in vitro models were developed based on neural progenitor cells from postmortem human neural tissues [27]. However, the access to these tissues is limited and the lifespan of the cultures is short [26].

With the emergence of human embryonic stem cells (hESCs) [28] and induced pluripotent stem cells (iPSCs) [29], there was a major breakthrough in new in vitro models for CNS developmental disorders. For example, following differentiation protocols, patient-derived iPSCs can be used as a platform to understand the molecular mechanisms behind a specific disease. Moreover, iPSCderived models can also serve as templates for drug-screening experiments, as well as other therapeutic strategies. For example, iPSCs obtained from patients with Costello syndrome (CS) [30] were differentiated towards a neuroectodermal fate and revealed an extended progenitor phase compared with controls, which resulted in an increased production of cortical neurons with altered neurite length and soma size. These changes have been described as typical of CS [30]. Another study used PSCs derived from patients with Down's syndrome to test chromosome-silencing therapies [31]. Deficits of proliferation and neural rosette formation were reversed, which opens a new door for potential 'chromosome therapy' techniques. This relatively new field of science aims, through the use of molecular biology-based techniques, to inactivate the chromosome that has been identified as the cause for a certain developmental disease (e.g., Down's syndrome). iPSC strategies have also been applied to the study of Rett syndrome [32] and autism spectrum disorders [33]. Using human iPSCs, Lancaster and coworkers [34] developed an in vitro brain model that could be used to mimic different developmental CNS disorders as well as other brain diseases. The so-called 'cerebral organoids' were capable of forming different interdependent brain regions, demonstrating several hallmarks of brain development. However, the main limitation of this model is the lack of a vascular system for nutrients and oxygen exchange. In a disease context, this model proved to be useful for the study of microcephaly and some of its molecular basis. This is of interest given the difficulties in recapitulating this condition using animal models.

When traumatic injuries occur in the CNS, nervous tissue damage is usually irreversible. The biological and cellular processes involved in nerve degeneration are currently not fully understood, which might be due, in part, to the complexity of the mechanisms underlying such injuries. Advances in the investigation of in vitro models of nerve regeneration over the past few years have led to the development of valuable tools to study the neurobiology and neuroregeneration of the CNS, with the aim of establishing new therapies. Recently, CNS models have been developed using microplatforms that are designed to mimic the dynamics between several CNS cell types, such as neurons and glia, and the ECM $[35,36]$. Microfluidics and biomaterial technologies have been used to control the physical and chemical cues in such microenvironments. Microfluidic devices enable a connection to be formed between different chambers with the controlled insertion of fluids within it [37]. Such devices allow the study of communication between different cells and to monitor events such as axonal migration, synaptic activity, and neuronal networks. These devices are used to not only study nerve damage at a chemical and physical level of injury, but also to recreate the postinjury environment [35]. Biomaterials are also used to control the in vitro CNS microenvironment by using several patterning techniques, such as lithography [38] and electrospinning [39], usually with surface modifications with ECM-like molecules and synthetic peptides $[40,41]$ to enhance their bioactivity. For example, Silva et al. demonstrated that gellan gum-based hydrogels modified with fibronectin-derived peptides were able to increase both NSC [42] and bone marrow mesenchymal stem cells (MSCs) [43] metabolic activity, proliferation, and differentiation. Given that cellular behavior is highly dependent on the surrounding microenvironment, the presence of biological cues in patterned surfaces are crucial for cell responsiveness and interaction with the microenvironment [40].

Another interesting example of the use of bioengineered systems is in the study of developmental neurotoxicity (DNT). Malformations and developmental retardation are a major health 
concern associated with environmental and consumer-product chemicals, for which there have been no effective tools of study. The use of 3D neural tissue-like constructs with fully matured neuronal networks (derived from NSC obtained from CNS tissue or from PSCs) growing on hydrogel-based biomaterials, interfacing with microelectrode arrays for electrical stimulation and response recording, were proposed as a model of study [44]. In these systems, the hydrogels act as a surrogate of the ECM found in vivo. By grafting peptides and/growth factors typical of the ECM of the cell, it is possible to recapitulate its niche, as well as the interactions between stem and fully differentiated cells with that niche. Moreover, hydrogels typically have mechanical properties similar to those of the CNS ECM, which facilitate cell growth, migration, and axonal elongation $[42,43]$. By contrast, during neuronal differentiation, several parameters, including $\mathrm{pH}$, cell growth, biomarkers of cell death, or even the establishment of action potentials, could be screened while the cells are exposed to toxic compounds, by using the microelectrode systems. These read outs could then establish a correlation between the conditions that were tested, their functional outputs, and the possible signaling and/or molecular pathways involved. Buzanska and colleagues [45] also suggested the use of cell growth platforms based on NSC and bioactive bioengineered surfaces obtained by microcontact printing or piezoelectric spotting of polycationic biomolecules and/or ECM proteins on cell-repellent surfaces. The rationale for this was to mimic the natural microenvironment of NSCs during development and predict the action of several compounds on DNT. Such a system could also be coupled with microelectrode arrays to determine the functionality of the cells being assessed. Thus, this multiparametric assessment is an obvious advantage because information on the sensitivity of certain molecular pathways and functional cellular responses to selected neurotoxins can be rapidly screened.

\section{Neurodegeneration}

Neurodegenerative processes within the CNS represent insults against the homeostasis of the CNS, capable of promoting cell death in neural populations in the brain and spinal cord [46]. In recent years, several different bioengineered systems have been developed to either study degeneration itself or, alternatively, routes to overcome it either through neuroprotection or neurodifferentiation-based studies. These systems range from compartmentalized chambers, to hydrogel-based biomaterials or even microfluidics-based approaches.

For instance, Piscioneri et al. [47] described the development of a compartmentalized membrane system using neonatal rodent hippocampal cells and human MSCs (hMSCs) to investigate the neuroprotective effects of the latter in a oxygen-glucose deprivation (OGD) model. The goal was to simulate cerebral ischemic damage by inducing OGD for $120 \mathrm{~min}$. Cells were cultured in a membrane system with a sandwich configuration in which the hippocampal cells were seeded on a fluorocarbon (FC) membrane, and were separated by hMSCs through a semipermeable polyethersulfone (PES) membrane that ensured the transport of paracrine factors, but prevented cell-to-cell contact. With this interesting system, the authors were able to show that the hMSC secretome protected hippocampal cells against the OGD insult.
In addition to these systems, recent developments in 3D cellular culture systems have been made to study the onset of pathogenesis and early development of a disease. Kim and colleagues [48] recently developed the first human 3D cellular culture system through the use of human neural progenitors (hNPCs), able to recreate the key events of $\mathrm{AD}$ pathogenesis, such as the accumulation of amyloid- $\beta$ (A $\beta)$ and hyperphosphorylated tau. In this particular system, hNPCs that produce high concentrations of pathogenic $A \beta$ species are combined with a Matrigel-based 3D culture system, providing an environment that favors $A \beta$ deposition. According to the authors, the culture system takes $1-2$ days to establish, while $A \beta$ is formed after 6 weeks of differentiation and Tau-related pathology after 10-14 weeks. This kind of approach could provide a flexible scalability that could make it an ideal model for application to other neurodegenerative disorders (e.g., PD), being not only suitable for the study of diseases characterized by abnormal aggregation of misfolded proteins, but also for new diagnostic and/or prognostic biomarkers, large-scale testing, and drug screenings $[48,49]$.

Finally, by using 3D bioprinting, Hsiesh and Hsu [50] embedded NSCs in a thermoresponsive biodegradable polyurethane (PU) bioink, creating a printed 3D cell culture system with robust levels of viability and differentiation after printing that could be further used in the context of neurodegenerative disease. Low-cost 3D printed devices have also been developed for growing and imaging primary neuronal cultures, revealing the multiple uses of this technology [51]. Indeed, 3D printing techniques could represent a fast and reliable method for the establishment of 3D organoidbased models for CNS research [52,53]. Cell and/or biomaterial constructs with a layer-by-layer architecture enable the separation of different cell types that can still interact via paracrine action, mimicking, for instance, some of the phenomena seen in brain barriers or trauma-related conditions. Additionally, each of these layers can have its own specific properties (e.g., ECM motifs, rigidity, or diffusion) enabling from the very beginning (because the system allows cells to be embedded while biomaterials are being printed) the creation of different subniches under the same cell culture setup paradigm. Finally, although these models do currently represent an additional cost compared with standard 2D cell-based models, the outputs obtained from them, as well as their proximity to in vivo environments, make them viable for the applications discussed here. Moreover, it is also expected that the costs associated will decrease with the further development of the technology.

\section{Trauma}

SCI and TBI are leading causes of death and disabilities worldwide $[48,54]$. These injuries are usually caused by external physical insults, most frequently as a result of impact, such as contusion and compression, or due to laceration, resulting in severe functional disabilities [54]. Subsequent to the initial trauma, a wellcharacterized cascade of events, including necrosis, hemorrhage, oxidative stress, excitotoxicity, inflammation, cavity formation (e.g., spinal cord), and astrogliosis, occurs in both SCI and TBI that further compromises neurologic function.

In vitro models of trauma in the $\mathrm{CNS}$ are obtained using different mechanical stimuli, either by using basic 2D or more complex 3D culture systems. The most common are transection (by lacerating 

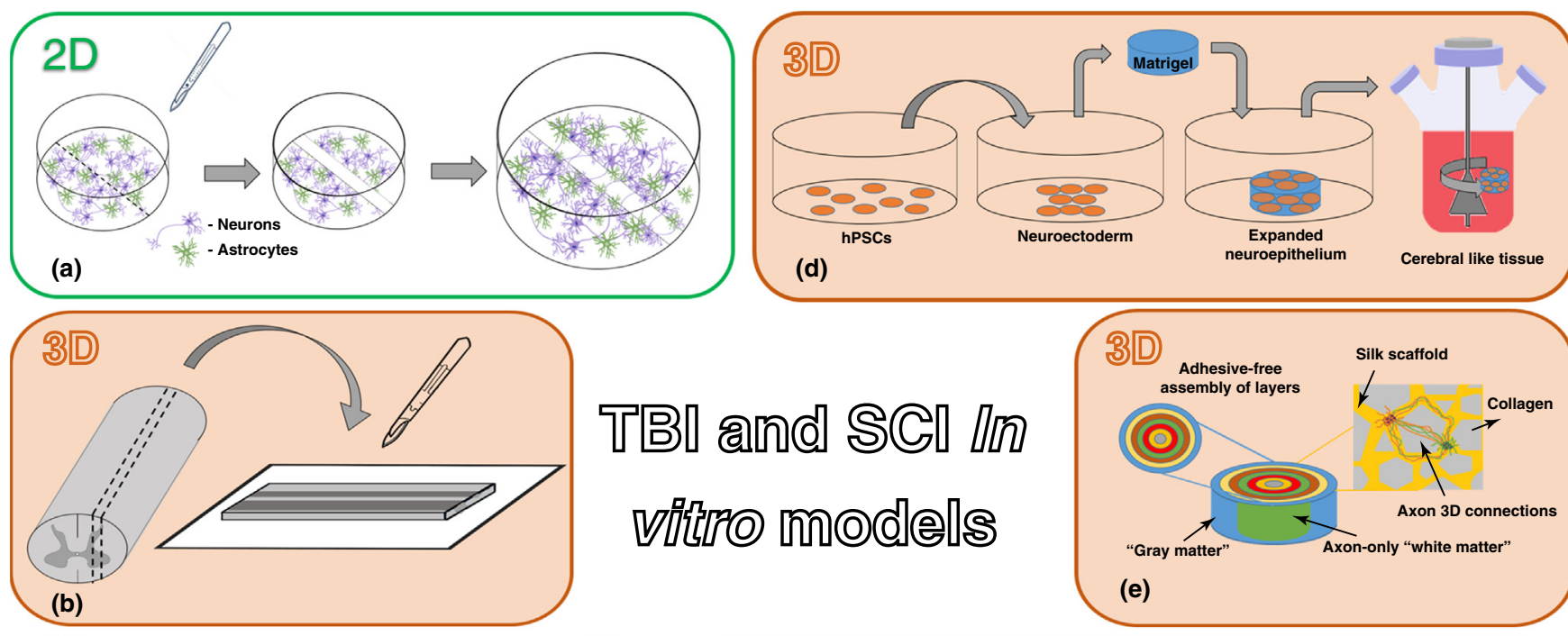

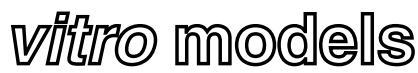
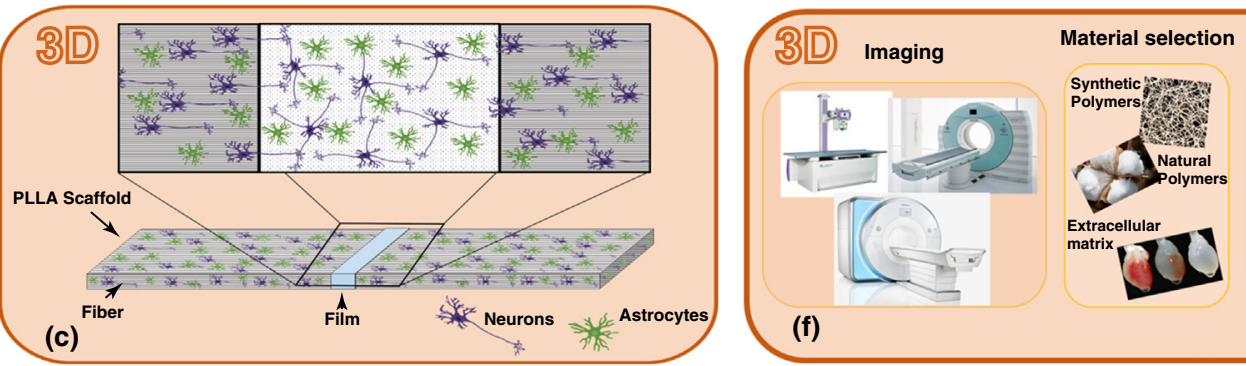

(e)
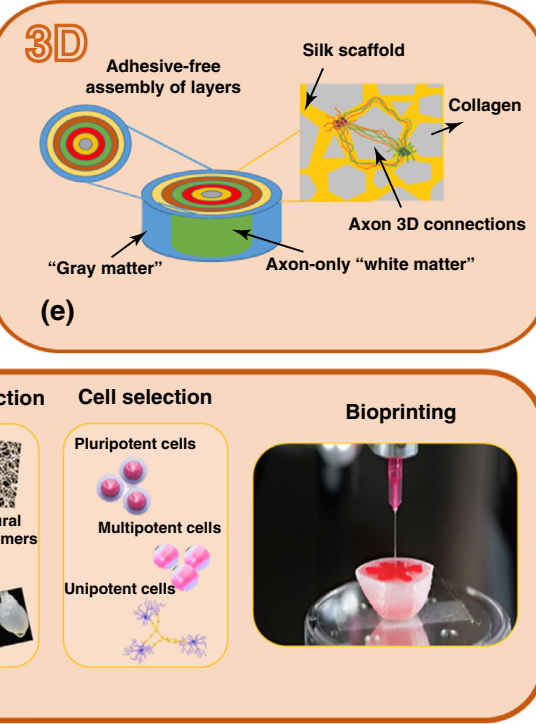

$\overline{\text { Drug Discovery Today }}$

\section{FIGURE}

Schematic representation of traumatic brain injury (TBI) and spinal cord injury $(\mathrm{SCl})$ in vitro models $(\mathrm{a}) \mathrm{SCl} 2 \mathrm{D}$ in vitro model from spinal cord embryo cells. After cutting the monolayer, an initial cell-free area appears, accompanied over time by many features of SCI [58]. (b) In vitro production and lesioning of organotypic spinal cord slice cultures [59]. (c) Sterilized poly-L-lactide (PLLA) fiber scaffolds coated with fibronectin are used to culture both neurons and astrocytes, forming a neuronal 3D in vitro model [60]. (d) Schematic representation of each stage of the culture system methods to obtain a 3D in vitro cerebral-like tissue [34]. (e) Design concept of an in vitro cortical tissue. The material design of the scaffold composite supports the 3D axon connections. The resulting module comprises two regions: neuron-rich gray matter and axon-only matter [64]. (f) Bioprinting: imaging and assessment of the damaged tissue and its environment are first performed, and then the most suitable material and cell type are selected [52]. Both cells and material can be plotted at same time using new rapid prototyping equipment. Some tissues require a period of maturation in a bioreactor before transplantation.

or cutting axons in primary neuronal or organotypical cultures) or compression (mechanical damage, usually by manually crushing CNS structures, commonly in organotypical cultures). Other systems try to tackle more complex insults, such as drastic acceleration and deceleration processes, which can cause, for instance, diffuse axonal injury or barotrauma, which can result from pressure waves that propagate through the intracranial and/or spinal contents as a consequence of trauma.

Injury to $2 \mathrm{D}$ cultures can be achieved by applying one or more injury-related stimuli [55]. For example, Jowers used a scratchinduced method of injury to model TBI, whereas Salvador and colleagues used stretch injury in combination with OGD to mimic ischemic events that occur after TBI $[56,57]$. By contrast, to create a SCI in vitro model, Boomkamp and colleagues cultured dissociated rat embryonic spinal cord cells plated onto a monolayer of astrocytes. After cutting the cultures, an initial cell-free area appeared devoid of neurites, accompanied over time by many features of SCI, including demyelination, reduced neurite density adjacent to the lesion, and infiltration of microglia and reactive astrocytes into the lesioned area. The pharmacological manipulation of this system using Rho and ROCK inhibitors revealed that both neurite extension and myelination could be observed in the initial cellfree area, showing the validity of the model as a tool to predict axonal growth in a SCI-like environment. (Fig. 1a) [58].

$2 \mathrm{D}$ in vitro cultures of neuronal cells offer simplified highthroughput systems. However, they lack endogenous 3D cell-cell interactions and physiological cues provided by the ECM. Thus, organotypic experimental preparations, such as brain and spinal cord slices, have been used as tools to develop bioengineered systems in combination with biomaterials. An interesting ex vivo SCI model using longitudinal spinal cord sections (Fig. 1b) was developed by Weightman and colleagues to test the viability of biomaterial platforms allowing high-throughput screening of neuroregenerative biomaterials [59]. In turn, biomaterials can also be used to model the injury site. Recently, an in vitro culture system using patterned electrospun fibers of poly-L-lactic acid and smooth films was developed to study how astrocytes respond to local changes in surface topography that might be similar to topographical changes following SCI (Fig. 1c) [60].

The previously referred to organoid-based culture systems could also become valuable tools for SCI and TBI research (Fig. 1d). However, these systems have limitations, such as the degree of 
maturation and absence of a vascular system [34,61]. Indeed, the vascular system is important under the context of a trauma-based response following CNS injury. Through the reorganization of the vasculature and angiogenesis, both oxygen and growth factor gradients can be established, leading to chemotactic responses by host cells involved in key processes, such as cell survival, differentiation, and inflammation. For instance, Duah and colleagues recently showed how angiogenesis could be related to the improved functionality of a rat SCI animal model, as a response to a neurotrophin-3 (NT-3)-based therapy [62].

Similarly to other conditions, tissue-engineered constructs can also be used for CNS trauma-related studies. Odawara and colleagues developed 3D neuronal networks with a layered structure using collagen fibers and polydimethylsiloxane microchambers [63]. Tang-Schomer and colleagues went a step further in developing functional, long-term viable, 3D brain-like cortical tissues, with gray and white matter compartmentalization and electrophysiological function using silk protein-based scaffolds combined with a collagen gel (Fig. 1e). When a weight drop injury model was used, the tissue exhibited injury-induced glutamate release and transient electrical hyperactivity, recapitulating the in vivo pathophysiology of TBI [64].

\section{Concluding remarks}

Since their initial development at the start of the 20th century, cell culture systems have enabled scientists to better understand the physiology, biochemistry, and molecular mechanisms of almost all organs and systems within the human body, including the CNS. Given that the limitations of standard 2D cell cultures techniques were reached several years ago, bioengineered 3D systems are presented as the alternative for the further development of this technical field. With a multiparameter analysis always present, these systems, based on hydrogels, membranes, microfluidics, stem cells, and microelectrode arrays, enable researchers to not only culture cells in environments that mimic the different CNS niches, but also obtain important data on the functionality of the CNS cells that are being used. However, such systems still have several disadvantages, particularly their complexity, which usually demands specific expertise, and the cost, which is currently significantly higher compared with standard 2D systems. Nevertheless, their potential is high and, therefore, they should be considered as the future of in vitro studies in CNS research.

\section{Acknowledgments}

Financial support is acknowledged from Prémios Santa Casa Neurociências - Prize Melo e Castro for Spinal Cord Injury Research; Portuguese Foundation for Science and Technology [Doctoral fellowship (SFRH/BD/103075/2014) to E.D.G.; IF Development Grant to A.J.S.; Starting Grant to F. Marques; PostDoctoral fellowship SFRH/BPD/97701/2013 to N.A.S.]; this work was co-funded by Programa Operacional Regional do Norte (ON.2 - O Novo Norte), ao abrigo do Quadro de Referência Estratégico Nacional (QREN), através do Fundo Europeu de Desenvolvimento Regional (FEDER).

\section{References}

1 Hartung, T. (2013) Food for thought; look back in anger - what clinical studies tell us about preclinical work. ALTEX 30, 275-291

2 Lowenstein, P.R. and Castro, M.G. (2009) Uncertainty in the translation of preclinical experiments to clinical trials. Why do most phase III clinical trials fail? Curr. Gene Ther. 9, 368-374

3 Pires, A.O. et al. (2014) The secretome of bone marrow and Wharton jelly derived mesenchymal stem cells induces differentiation and neurite outgrowth in SH-SY5Y cells. Stem Cells Int. 438352

4 Li, W. et al. (2015) Folic acid methylation profile of JAK-STAT and long-term depression signaling pathways in Alzheimer's disease models. Mol. Neurobiol. http://dx.doi.org/10.1007/s12035-015-9556-9 Published online December 1, 2015

5 Joshi, M.D. et al. (2016) Evaluation of liposomal nanocarriers loaded with ETB receptor agonist, IRL-1620, using cell-based assays. Neuroscience 312, 141-152

6 Silva, N.A. et al. (2010) Development and characterization of a novel hybrid tissue engineering-based scaffolds for spinal cord injury repair. Tissue Eng. Part A 16, 45-54

7 Hopkins, A.M. et al. (2015) 3D in vitro modeling of the central nervous system. Progr. Neurobiol. 125, 1-25

8 Gonzalez-Perez, F. et al. (2015) Substratum preferences of motor and sensory neurons in postnatal and adult rats. Eur. J. Neurosci. 43, 431-442

9 Saito, S. et al. (2016) An in vitro reproduction of stress-induced memory defects: effects of corticoids on dendritic spine dynamics. Sci. Rep. 6, 19287

10 Pohland, M. et al. (2015) Studying axonal outgrowth and regeneration of the corticospinal tract in organotypic slice cultures. J. Neurotrauma 32, 1465

11 Heine, C. and Franke, H. (2014) Organotypic slice co-culture systems to study axon regeneration in the dopaminergic system ex vivo. Meth. Mol. Biol. 1162, 97-111

12 Abbott, N.J. (2005) Dynamics of CNS barriers: evolution, differentiation, and modulation. Cell. Mol. Neurobiol. 25, 5-23

13 Ballabh, P. et al. (2004) The blood-brain barrier: an overview: structure, regulation, and clinical implications. Neurobiol. Dis. 16, 1-13

14 Redzic, Z.B. and Segal, M.B. (2004) The structure of the choroid plexus and the physiology of the choroid plexus epithelium. Adv. Drug Deliv. Rev. 56, 1695-1716

15 Marques, F. et al. (2009) Altered iron metabolism is part of the choroid plexus response to peripheral inflammation. Endocrinology 150, 2822-2828

16 Nicolazzo, J.A. et al. (2006) Methods to assess drug permeability across the bloodbrain barrier. J. Pharm. Pharmacol. 58, 281-293

17 Laschinger, M. and Engelhardt, B. (2000) Interaction of $\alpha 4$-integrin with VCAM- 1 is involved in adhesion of encephalitogenic $\mathrm{T}$ cell blasts to brain endothelium but not in their transendothelial migration in vitro. J. Neuroimmunol. 102, 32-43

18 Bicker, J. et al. (2014) Blood-brain barrier models and their relevance for a successful development of CNS drug delivery systems: a review. Eur. J. Pharm. Biopharm. 87, 409-432

19 Mesquita, S.D. et al. (2014) Lipocalin 2 modulates the cellular response to amyloid beta. Cell Death Diff. 21, 1-12

20 Yarong, H. et al. (2014) Cell culture models of the blood brain barrier. Stroke 45, 2514-2526

21 Stanness, K.A. et al. (1996) A dynamic model of the blood brain barrier 'in vitro'. Neurotoxicology 17 481-196

22 Santaguida, S. et al. (2006) Side by side comparison between dynamic versus static models of blood brain barrier in vitro: a permeability study. Brain Res. 1109, 1-13

23 Cucullo, L. et al. (2007) Development of a humanized in vitro blood-brain barrier model to screen for brain penetration of antiepileptic drugs. Epilepsia 48, 505-516

24 Alcendor, D.J. et al. (2013) Neurovascular unit on a chip: implications for translational applications. Stem Cell Res. Ther. 4, 1

25 Brown, J.A. et al. (2015) Recreating blood-brain barrier physiology and structure on chip: a novel neurovascular microfluidic bioreactor. Biomicrofluidics 9, 054124

26 Telias, M. and Ben-Yosef, D. (2014) Modeling neurodevelopmental disorders using human pluripotent stem cells. Stem Cell Rev. 10, 494-511

27 Verwer, R.W. et al. (2002) Cells in human postmortem brain tissue slices remain alive for several weeks in culture. FASEB J. 16, 54-60

28 Thomson, J.A. et al. (1998) Embryonic stem cell lines derived from human blastocysts. Science 282, 1145-1147

29 Takahashi, K. et al. (2007) Induction of pluripotent stem cells from adult human fibroblasts by defined factors. Cell 131, 861-872

30 Rooney, G.E. et al. (2016) Human iPS cell-derived neurons uncover the impact of increased Ras signaling in Costello syndrome. J. Neurosci. 36, 142-152

31 Jiang, J. et al. (2013) Translating dosage compensation to trisomy 21. Nature 500, 296-300 
32 Ricciardi, S. et al. (2012) CDKL5 ensures excitatory synapse stability by reinforcing NGL-1-PSD95 interaction in the postsynaptic compartment and is impaired in patient iPSC-derived neurons. Nat. Cell Biol. 14, 911-923

33 Lin, M. et al. (2011) RNA-Seq of human neurons derived from iPS cells reveals candidate long non-coding RNAs involved in neurogenesis and neuropsychiatric disorders. PLOS ONE 6, e23356

34 Lancaster, M.A. et al. (2013) Cerebral organoids model human brain development and microcephaly. Nature 501, 373-379

35 Siddique, R. and Thakor, N. (2014) Investigation of nerve injury through microfluidic devices. J. R. Soc. Interface 11, 20130676

36 Yi, Y. et al. (2015) Central nervous system and its disease models on a chip. Trends Biotechnol. 33, 762-776

37 Mills, C.A. et al. (2007) Nanoembossed polymer substrates for biomedical surface interaction studies. J. Nanosci. Nanotechnol. 7, 4588-4594

38 Yang, K. et al. (2015) Biodegradable nanotopography combined with neurotrophic signals enhances contact guidance and neuronal differentiation of human neural stem cells. Macromol. Biosci. 15, 1348-1356

39 Yang, F. et al. (2005) Electrospinning of nano/micro scale poly(L-lactic acid) aligned fibers and their potential in neural tissue engineering. Biomaterials 26, 2603-2610

40 Nakanishi, J. et al. (2008) Recent advances in cell micropatterning techniques for bioanalytical and biomedical sciences. Anal. Sci. 24, 67-72

41 Falconnet, D. et al. (2006) Surface engineering approaches to micropattern surfaces for cell based assays. Biomaterials 27, 3044-3063

42 Silva, N.A. et al. (2012) The effects of peptide modified gellan gum and olfactory ensheathing glia cells on neural stem/progenitor cell fate. Biomaterials 33, 6345-6354

43 Silva, N.A. et al. (2013) Modulation of bone marrow mesenchymal stem cell secretome by ECM-like hydrogels. Biochimie 95, 2314-2319

44 Bal-Price, A.K. et al. (2010) In vitro developmental neurotoxicity (DNT) testing: relevant models and endpoints. NeuroToxicology 31, 545-554

45 Buzanska, L. et al. (2010) Neural stem cells from human cord blood on bioengineered surfaces: novel approach to multiparameter bio-tests. Toxicology 270, $35-42$

46 Teixeira, F.G. et al. (2013) Mesenchymal stem cells secretome: a new paradigm for central nervous system regeneration? Cell. Mol. Life Sci. 70, 3871-3882

47 Piscioneri, A. et al. (2015) Neuroprotective effect of human mesenchymal stem cells ina compartmentalized neuronal membrane system. Acta Biomat. 24, 297-308

48 Kim, Y.H. et al. (2015) A 3D human neural cell culture system for modeling Alzheimer's disease. Nat. Protoc. 10, 985-1006
49 Choi, S.H. et al. (2014) A three-dimensional human neural cell culture model of Alzheimer's disease. Nature 515, 274-278

50 Hsieh, F.Y. et al. (2015) 3D bioprinting of neural stem cell-laden thermoresponsive biodegradable polyurethane hydrogel and potential in central nervous system repair. Biomaterials 71, 48-57

51 Wardyn, J.D. et al. (2015) Low cost production of 3D-printed devices and electrostimulation chambers for the culture of primary neurons. J. Neurosci. Meth. $15,17-23$

52 Murphy, S.V. and Atala, A. (2014) 3D bioprinting of tissues and organs. Nat. Biotechnol. 32, 773-785

53 Horváth, L. et al. (2015) Engineering an in vitro air-blood barrier by 3D bioprinting. Sci. Rep. 5, 7974

54 Rubiano, A.M. et al. (2015) Global neurotrauma research challenges and opportunities. Nature 527, S193-S197

55 Kumaria, A. and Tolias, C. (2008) In vitro models of neurotrauma. Br. J. Neurosurg. 22, 200-206

56 Jowers, C.T. et al. (2013) The cell injury device: a high-throughput platform for traumatic brain injury research. J. Neurosci. Meth. 218, 1-8

57 Salvador, E. et al. (2015) Stretch and/or oxygen glucose deprivation (OGD) in an in vitro traumatic brain injury (TBI) model induces calcium alteration and inflammatory cascade. Front. Cell. Neurosci. 9, 323

58 Boomkamp, S.D. et al. (2012) The development of a rat in vitro model of spinal cord injury demonstrating the additive effects of Rho and ROCK inhibitors on neurite outgrowth and myelination. Glia 60, 441-456

59 Weightman, A.P. et al. (2014) An in vitro spinal cord injury model to screen neuroregenerative materials. Biomaterials 35, 3756-3765

60 Zuidema, J.M. et al. (2015) Nebulized solvent ablation of aligned PLLA fibers for the study of neurite response to anisotropic-to-isotropic fiber/film transition (AFFT) boundaries in astrocyte-neuron co-cultures. Biomaterials 46, 82-94

61 Meinhardt, A. et al. (2014) 3D reconstitution of the patterned neural tube from embryonic stem cells. Stem Cell Rep. 3, 987-999

62 Duan, H. et al. (2015) Transcriptome analyses reveal molecular mechanisms underlying functional recovery after spinal cord injury. Proc. Natl. Acad. Sci. U. S. A. $112,13360-13365$

63 Odawara, A. et al. (2013) A three-dimensional neuronal culture technique that controls the direction of neurite elongation and the position of soma to mimic the layered structure of the brain. RSC Adv. 3, 23620-23630

64 Tang-Schomer, M.D. et al. (2014) Bioengineered functional brain-like cortical tissue. Proc. Natl. Acad. Sci. U. S. A. 111, 13811-13816 